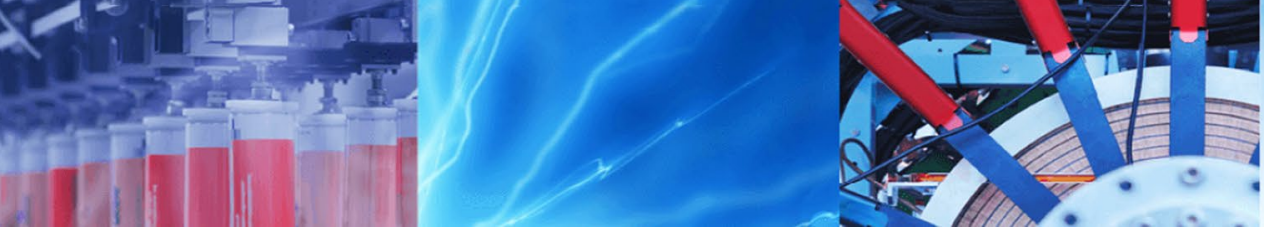

Research Article

\title{
Numerical, electronic simulations and experimental analysis of a no-equilibrium point chaotic circuit with offset boosting and partial amplitude control
}

\author{
Justin Roger Mboupda Pone ${ }^{1,2}$ (D) Victor Kamdoum Tamba ${ }^{3}$ Guillaume Honore Kom ${ }^{1,2}$. \\ Mathieu Jean Pierre Pesdjock ${ }^{1}$. Alain Tiedeu ${ }^{4} \cdot$ Martin Kom $^{2}$
}

(c) Springer Nature Switzerland AG 2019

\begin{abstract}
This paper investigates numerically and experimentally an electronic circuit modelling the dynamics of a system without equilibrium point recently introduced by Shahzad et al. (Eur Phys J Spec Top 224:1637-1652, 2015). By varying the parameters of the electronic circuit, new behaviors are found including antimonotonicity, chaotic bistable hidden attractors, chaotic bubble hidden attractors, offset boosting and partial amplitude control. Another outcome of this paper is the forward and reversed interior crisis features found in a chaotic circuit without equilibrium point. Laboratory experiments and PSIM simulations are carried out to confirm results predicted by numerical simulations.
\end{abstract}

Keywords Chaotic circuit · Antimonotonicity - Chaotic hidden bubble attractors - Offset boosting and partial amplitude control · PSIM analog simulations · Laboratory electronic experiments

\section{Introduction}

In the last five decades, chaos as a very rich and interesting complicated nonlinear phenomena has been intensively studied. A system is said to be chaotic, if its sensitive to initial conditions and has an infinite number of unstable periodic trajectories of different periods. Chaotic systems have been widely applied in various fields, such as economy [42], biology [44], medical diagnosis [9, 23], neural network [48], image encryption [5], secure communication [7], mechanic [54] just to name a few. Recently, researchers have introduced a new classification of nonlinear systems in which they focused on two kinds of attractors: self-excited and hidden attractors. A self-excited attractor is defined as an attractor that the basin of attraction is excited from unstable equilibria [29]. From this point of view, most known nonlinear systems, such as Lorenz system, Rössler system [36], Chen system or Van der Pol oscillator [14], belong to chaotic systems with self-excited attractors [2]. In contrast, a few unusual systems such as those with an infinite number of equilibrium points, with stable equilibria, or without equilibrium belong to systems with hidden attractors $[25,30,31,35,38,51]$. They are called hidden attractors because their basin of attraction does not intersect with a small neighborhoods of equilibria $[1,28,29,37]$.

Although there exist a large number of published studies describing chaotic systems with a countable number of saddle equilibrium points, chaotic systems without equilibrium have been subjected to

$\triangle$ Justin Roger Mboupda Pone, mboupdapone00@gmail.com | ${ }^{1}$ Research Unit of Automation and Applied Computer (RU-AIA), Electrical Engineering Department of IUT-FV, University of Dschang, PO Box: 134, Bandjoun, Cameroon. ${ }^{2}$ Laboratoire de Genie Electrique, Mecatronique et Traitement du Signal (LGEMTS), Electrical Engineering Department of National Advanced School of Engineering, ENSP, University of Yaoundé I, PO Box: 8390, Yaoundé, Cameroon. ${ }^{3}$ Research Unit of Automation and Applied Computer (RU-AIA), Department of telecommunication and Networking Engineering of IUT-FV, University of Dschang, PO Box: 134, Bandjoun, Cameroon. ${ }^{4}$ Signal, Image and Systems Laboratory, ENSET Ebolowa, PO Box: 886, Ebolowa, Cameroon. 
considerable discussions recently [15, 24, 49, 50, 53]. Hidden attractors are important in engineering applications because they allow the understanding of some unexpected and potentially disastrous behaviors in structures like: nonlinear power supply, bridges, electrical transport lines, aircraft wing and so on [17]. Therefore, study their behaviors is of great importance in engineering. These systems are very challenging and their investigation may reveal several nonlinear phenomena. It is worth nothing to remark that systems without equilibrium points reported in the literature, show little knowledge about the special phenomena, such as bistability, interior crisis, amplitude and offset control or antimonotonicity. For these reasons, we set our goal to study a system without equilibrium points that could present these interesting properties. The first property is bistability which is a very important feature of nonlinear systems $[4,13,19,40,46]$ because they have potential engineering applications such as modelling memory in neural network $[56,3,26]$ or random bit generation [34]. The second feature is antimonotonicity phenomena which arises where periodic orbits are created and destroyed as a control parameter is varied. This latter one is common in nonlinear systems with equilibrium points such as Duffing, Van der pol oscillator or Chua circuit, but rarely occurs in systems without equilibrium points. The third phenomenon is the interior crisis which concerns the global stability of the system. When a chaotic attractor is globally stable and a parameter is varied, the attractor moves and can intersect with the basin boundary. The system loses its global stability. This phenomenon is also very common in nonlinear systems with equilibria but rarely reported in systems without equilibria $[12,20,21$, 27]. The reported phenomenon of interior crisis in this paper arise both in forward and reverse directions not yet illustrated in a system without equilibrium points as far as the knowledge of authors go. The last phenomenon is the offset boosting and partial amplitude control. This behavior can be implemented in the circuit. Therefore it can be used as a chaotic transmitting encoder of sensitive messages in an unsecured channel while adjusting the transmitted signal to the channel characteristics $[39,43]$. It could also be used to improve radio frequency conversion efficiency in rectifier circuits for efficient wireless power transmission [18].

As a result, deeper exploration of systems with hidden attractors is an interesting topic for engineering applications and academic significance, therefore should receive further attentions. Research on hidden attractors are still going on and the investigation of striking behaviors offers a great challenge. On this way, systematic search to find 3-D chaotic systems with quadratic nonlinearity and no-equilibrium point was performed by Sajad et al. [16]. They discovered 17 examples of such systems. Among them, NE8 is a specific example which derives from Sprott A system. It is a special case of Nose-Hoover [41,47] oscillator that describes fluid flow, solid diffusion viscosity or heat conduction in engineering system. Motivated by the above idea, this paper presents deeper analysis of the dynamics of the electronic modified model NE8 slightly studied by Shahzad et al. [45]. They reported twin coexisting hidden attractors and the synchronisation of two coupled of NE8 using robust adaptive sliding mode control method. Our objective for deeper investigation of this system, is to enrich the literature with a system, presenting no-equilibrium point and exhibiting more complex behaviors. To the best of our knowledge, there are only few electronic circuits exhibiting bistable hidden chaotic attractors with antimonotonicity, interior crisis, offset booting and partial amplitude control.

The rest of this paper is structured as follows. We describe the circuit, its state equations and its general properties in Sect. 2. Numerical analysis of the system in order to predict the behavior of the circuit are presented in Sect. 3. Section 4 deals with PSIM based simulations and experimental studies in order to validate numerical simulations. Some concluding remarks are presented in Sect. 5.

\section{Circuit description and modelling}

The schematic diagram and experimental printed circuit board of the suggested system without equilibrium point are presented in Fig. 1. The electronic circuit contains six linear operational amplifiers, three analog multipliers, twelve resistors and three capacitors.

The Kirchhoff's laws can be applied to the schematic diagram of Fig. 1a to obtain the following set of three coupled first order differential equations describing the dynamic of the system:

$$
\left\{\begin{array}{l}
R C \frac{d V x}{d t}=V y, \\
R C \frac{d V y}{d t}=-V x-\frac{R}{R_{3}} \frac{V y V z}{1 V}, \\
R C \frac{d V z}{d t}=\frac{V x V y}{1 V} \frac{R}{R_{4}}+\frac{(V x)^{2}}{1 V} \frac{R_{1}}{R_{4}}-\frac{1 V R_{2}}{R_{4}} .
\end{array}\right.
$$

For the following changes of the variables and parameters,

$$
\begin{aligned}
& x=\frac{V x}{1 V}, y=\frac{V y}{1 V}, z=\frac{V z}{1 V}, T_{1}=\frac{R_{1}}{R}, T_{2}=\frac{R_{2}}{R}, \\
& R_{3}=\frac{R}{10}, R_{4}=10 R, t=\tau R C,
\end{aligned}
$$

\section{SN Applied Sciences}




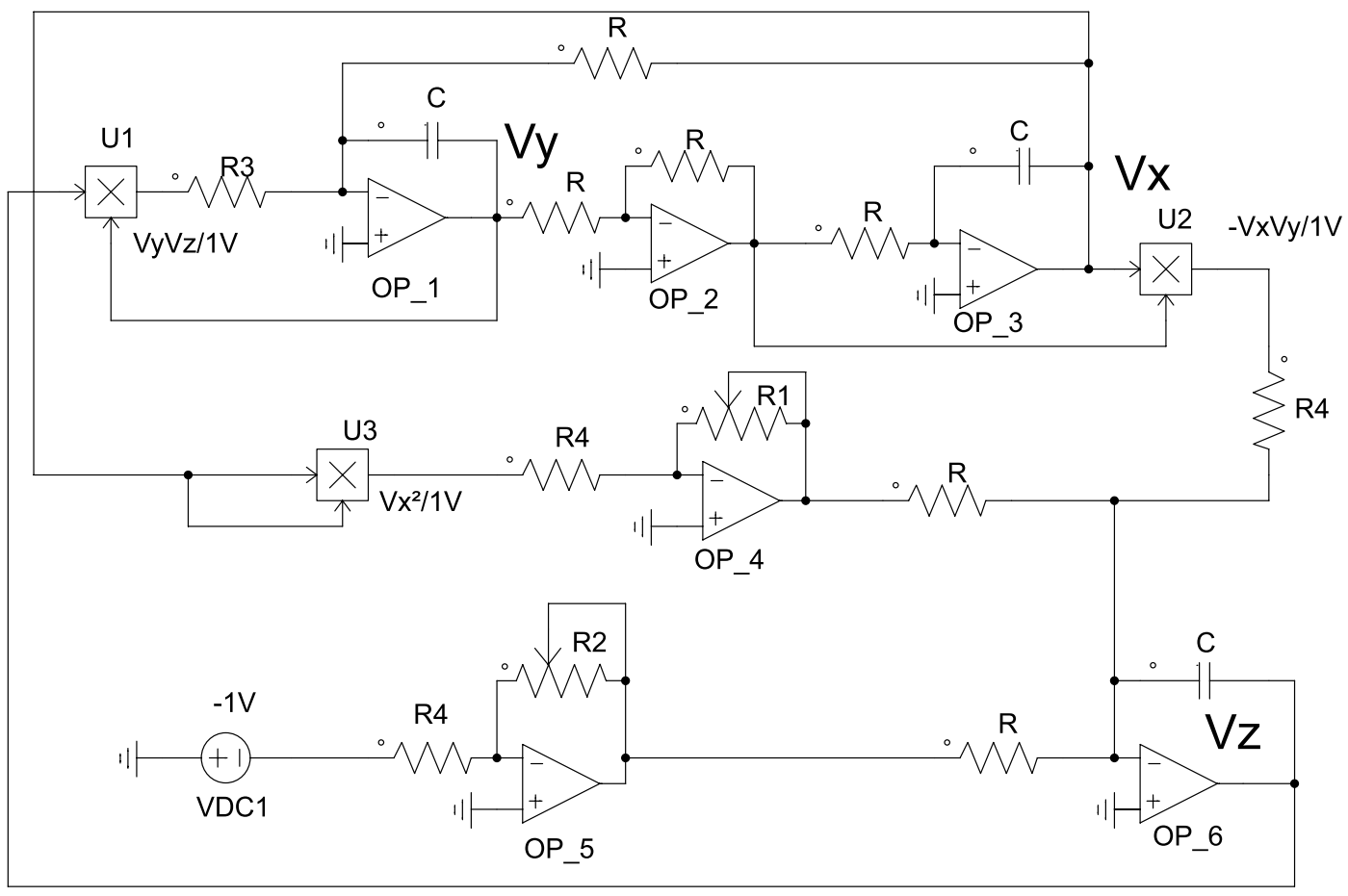

(a)

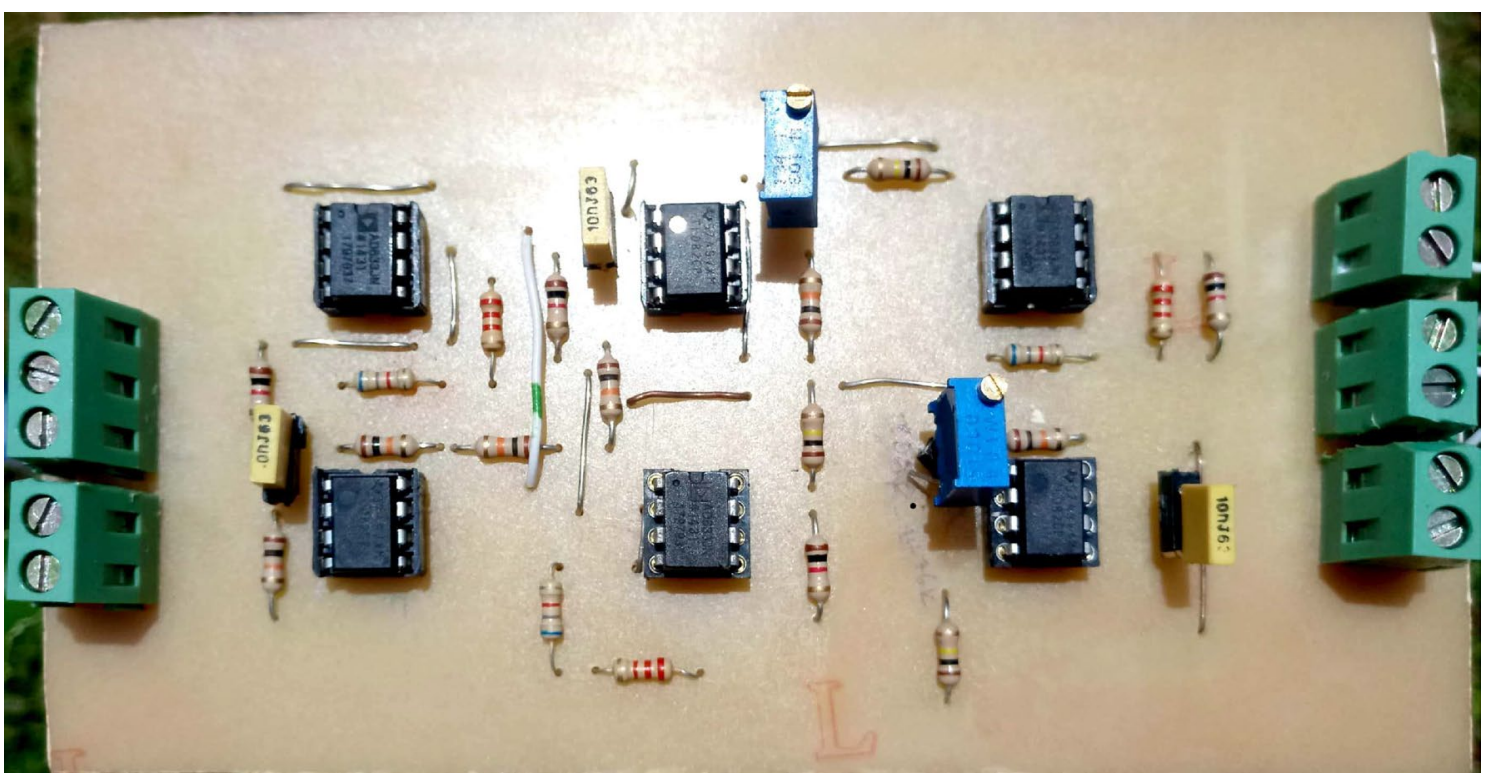

(b)

Fig. 1 a Electronic circuit diagram of the suggested system without equilibrium point (3) and $\mathbf{b}$ its experimental printed circuit board (PCB). The integrated circuits (IC) U1, U2, U3 are analog multipliers $A D 633 / J N$ and $O P_{1}-O P_{6}$ are operational amplifiers $T L 082$ operating in their linear regime. The IC are powered by a symmetric voltage source $\pm 15 \mathrm{~V} D C$. Capacitors $C$ are ceramic type of $10 \mathrm{nF}$ and $R$ are low power resistors of $10 \mathrm{k} \Omega$. The resistors $R_{4}=100 \mathrm{k} \Omega, R_{3}=1 \mathrm{k} \Omega$, the resistors $R_{2}$ and $R_{1}$ are variable resistors. The voltage $V_{D C 1}$ is an independent direct voltage source of $1 \mathrm{~V}$. (For the interpretation of the references to color in this figure, the reader is referred to the web version of this article) 
the set of Eq. (1) are expressed by the following nonlinear third-order differential equations:

$$
\left\{\begin{array}{l}
\dot{x}=y, \\
\dot{y}=-x-10 y z \\
\dot{z}=0.1 x y+0.1 T_{1} x^{2}-0.1 T_{2}
\end{array}\right.
$$

where the dot denotes differentiation with respect to the dimensionless time $\tau$. It is trivial to deduce the equilibrium points of system (3) by solving $\dot{x}=0 ; \dot{y}=0$; $\dot{z}=0$. This computation shows that there is no equilibrium point since $T_{2} \neq 0$. Therefore, the system under investigation belongs to the rare class of systems without equilibria. It is easy to notice that system (3) is invariant under the transformation: $(x, y, z) \Leftrightarrow(-x,-y, z)$. In the mathematical model (3), two parameters are identified $T_{1}$ and $T_{2}$. The modified system NE8 presented in Eq. (3) has two tuning parameters in contrary to one in Ref. [45] where the only controlled parameter is $T_{2}$. The second parameter allows us as it shall be seen in Sect. 3 to discover more nonlinear phenomena in contrast to only symmetric coexisting attractors reported in Ref. [45].

\section{Numerical studies}

The regions of various dynamical behaviors of system (3) as a function of two parameters $T_{1}$ and $T_{2}$ are shown in Fig. 2. It is noticed that chaotic bands (black color) are surrounded in upper limit by periodic behaviors (cyan color) and in lower limit (on the right side) by unbound behavior of the system (red color). We remark also some

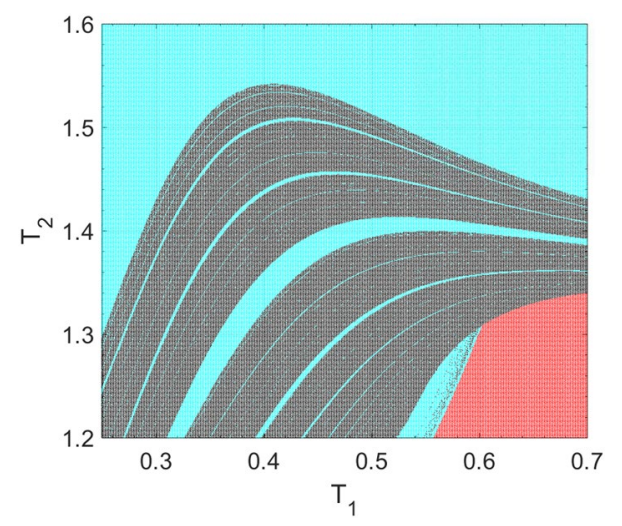

Fig. 2 The 2-parameters bifurcation diagram in plane $\left(T_{1}, T_{2}\right)$ depicting regions of various dynamical behaviors of system (3) including chaotic, periodic, and unbounded behaviors shown respectively in black, cyan and red. (For the interpretation of the references to color in this figure, the reader is referred to the web version of this article)

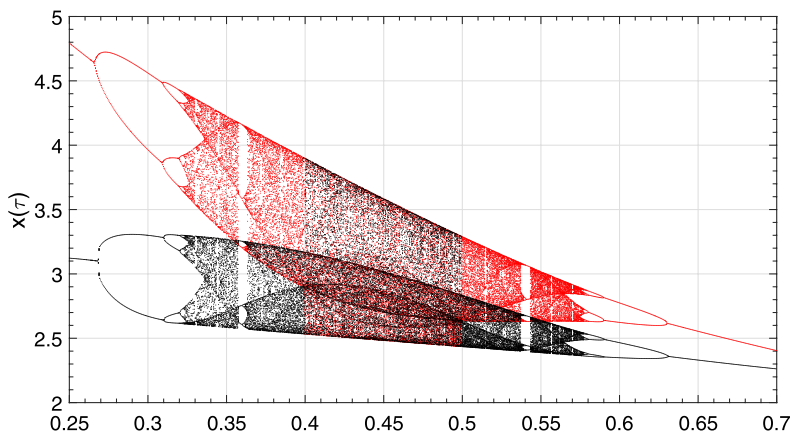

(a)

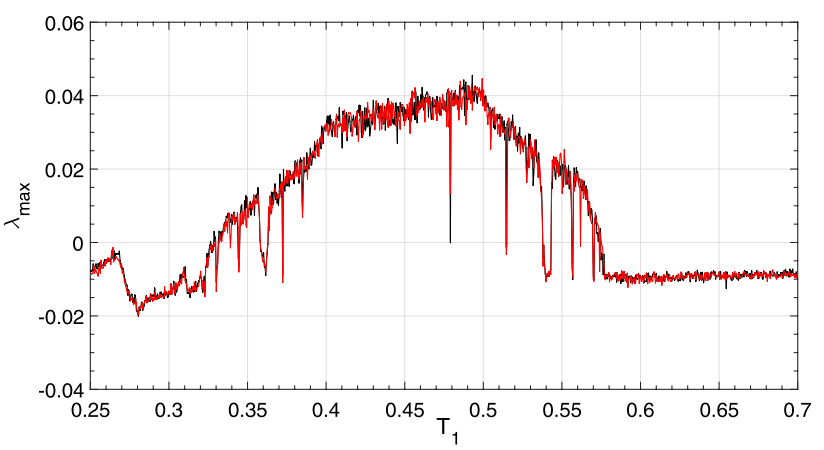

(b)

Fig. 3 Bifurcation diagrams (a) and corresponding graph of largest Lyapunov exponent (b) showing various behaviors of the system (3) for increasing parameter $T_{1}$ (black) and decreasing parameter $T_{1}$ (red); the parameter $T_{2}$ is fixed as $T_{2}=1.476$ and the initial conditions are chosen as $\left(x_{0} ; y_{0} ; z_{0}\right)=(0.1 ; 3.9 ; 0.1)$. (For the interpretation of the references to color in this figure, the reader is referred to the web version of this article)

tiny bands of periodic behaviors separating large band of chaotic behaviors.

\subsection{Period-doubling route to bistable chaos}

The bifurcation diagram of the state variable $x(\tau)$ and the corresponding Maximum Lyapunov exponent with respect to the parameter $T_{1}$ is presented in Fig. 3 .

The bifurcation diagram in Fig. 3a contains two sets of data: increasing (black) and decreasing (red) values of the control parameter $T_{1}$ superimposed.

This Bifurcation diagram shows that the system undergoes period-doubling in the range $0.25<T_{1}<0.35$; i.e. period- $1 \rightarrow$ period- $2 \rightarrow$ period $-4 \rightarrow$ period $-8 \rightarrow$ period $-16 \rightarrow \ldots$ chaos. It is noticeable that two bistable attractors exist in this interval of $T_{1}$. The two symmetric bistable chaotic attractors merge to form a unique chaotic attractor for $T_{1}$ in the interval $0.41<T_{1}<0.49$. Finally, the phenomenon of reverse period-doubling occurs for $T_{1}=0.57$. Within the interval $0.57<T_{1}<0.65$ reversed 

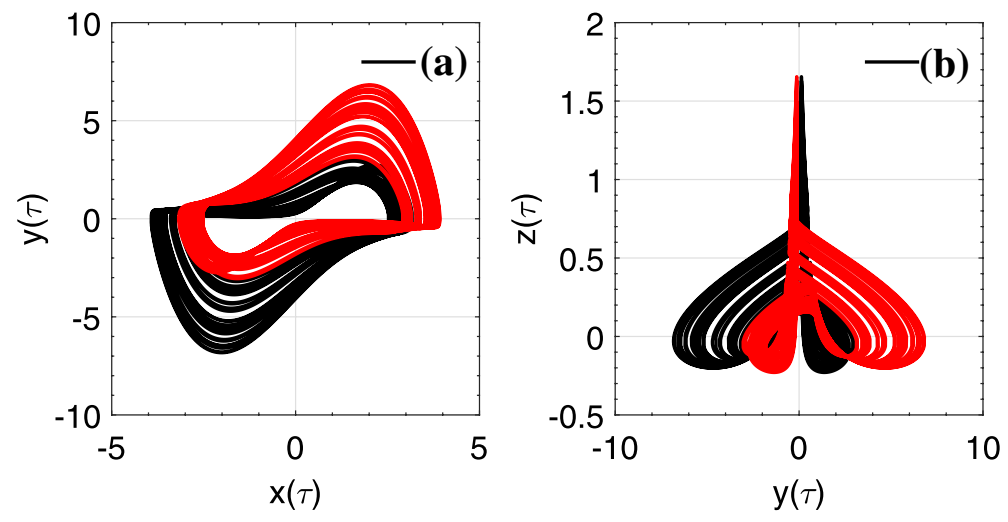

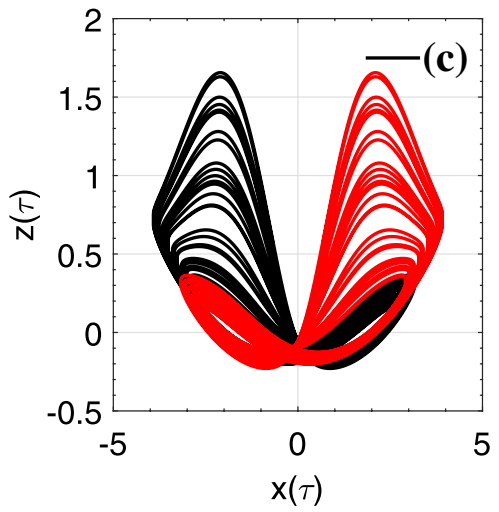

Fig. 4 2-D plots of numerical chaotic phase portraits showing bistability behavior of hidden attractors (red and black curves superimposed) in system (3) in the planes $\mathbf{a} x-y, \mathbf{b} y-z, \mathbf{c} x-z$ with $T_{2}=1.476$ and $T_{1}=0.395$. The initial conditions are fixed as

period-doubling continue in this pattern: chaos $\rightarrow \ldots$ period- $16 \rightarrow$ period- $8 \rightarrow$ period- $4 \rightarrow$ period $-2 \rightarrow$ period -1 . It can be seen that the bifurcation diagram coincides with the spectrum of the largest Lyapunov exponent (see Fig. 3b) which is computed numerically based on the algorithm described by Wolf et al. [55]. The phase portraits of bistable hidden attractors are shown in Fig. 4.

\subsection{Forward and reverse interior crisis}

The striking phenomenon of interior crisis was first discovered by Grebori et al. [10, 11]. It was described as a bifurcation event in which a chaotic attractor suddenly expands in size. This event occurs frequently in chaotic systems when the chaotic attractor collides with the unstable manifold of the equilibrium point as a parameter is varied. Figure 5 displays a zoom of Fig. 3 for $T_{1}$ in the range $0.38 \ll T_{1} \ll 0.52$.

In Fig. 5, one can observe that for $T_{1}<0.4$, the attractor consists of one distinct band which is developed from period-doubling cascade. At $T_{1}=T_{1-\text { low }}^{\text {critical }} \approx 0.3982$, the attractor suddenly expands and fill the previous avoided area illustrating the interior crisis phenomenon. By increasing $T_{1}$, this behavior remains until $T_{1} \approx 0.49$. At $T_{1}=T_{1-h i g h}^{\text {critical }} \approx 0.4997$, the attractor suddenly reduces and continues this reduction in size with the phenomenon of reverse period-doubling. This is typically the description of the reverse interior crisis event. Notice that the maximum Lyapunov exponent is the highest $\left(\lambda_{\max }>0.04\right)$ in the crisis interval $0.3982 \ll T_{1} \ll 0.4997$ revealing the high degree of chaos during crisis. (See Fig. 5b). This striking phenomena is confirmed by the phase portraits in Fig. 6(i) where one can easily depict $\left(x_{0} ; y_{0} ; z_{0}\right)=(0.1 ; \pm 3.9 ; 0.7)$. (For the interpretation of the references to color in this figure, the reader is referred to the web version of this article)

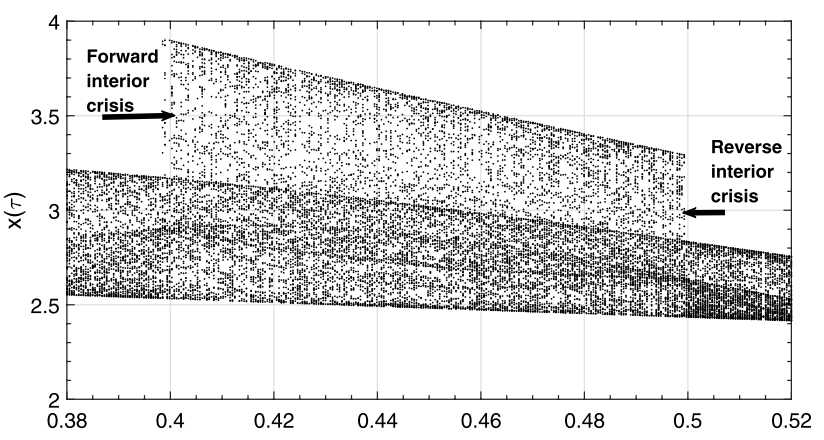

(a)

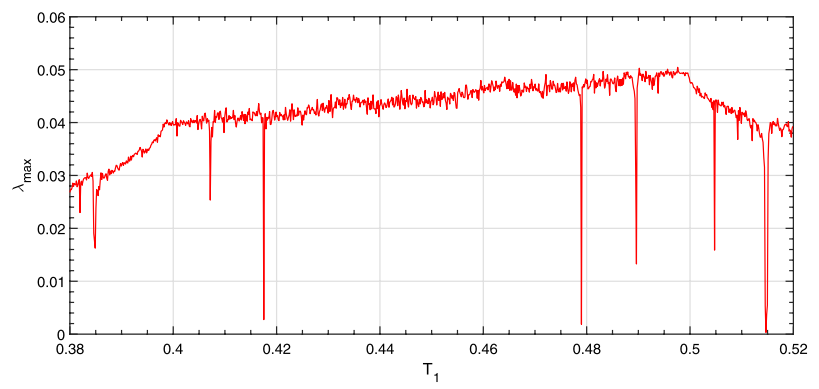

(b)

Fig. 5 Bifurcation diagram (a) and corresponding graph of the largest Lyapunov exponent (b) showing a zoom of the interior crisis intervals of $x(\tau)$ in Fig. 3 with respect to the parameter $T_{1} \cdot T_{2}$ is fixed as $T_{2}=1.476$. Forward interior crisis occurs at $T_{1-\text { critical }} \approx 0.3982$ and reverse interior crisis occurs at $T_{1-\text { high }}^{\text {critical }} \approx 0.4997$. Initial conditions are fixed as $(x 0 ; y 0 ; z 0)=(0.1 ; 0.1 ; 0.1)$

attractors before (Fig. 6a), during (Fig. 6b) and after (Fig. 6c) the interior crisis events.

The reader can notice abrupt change in the size of the attractor. The computed power density spectra in (6)(ii) 
Fig. 6 2-D projections of the attractors of system (3) in the $z-y$ plane (i) and the corresponding graph of the power density spectra (ii) illustrating crisis transitions: the transition from $(\mathbf{a}) \Rightarrow(\mathbf{b})$ indicates the forward interior crisis behavior; while the transition from $(\mathbf{b}) \Rightarrow$ (c) corresponds to reverse interior crisis phenomenon. The values of the parameters are selected as $T_{1}=0.395$ in (a), $T_{1}=0.49$ in (b) and $T_{1}=0.5$ in (c). $T_{2}$ is fixed as $T_{2}=1.476$ and the initial conditions are $\left(x_{0} ; y_{0} ; z_{0}\right)=(0.1 ; 0.1 ; 0.1)$
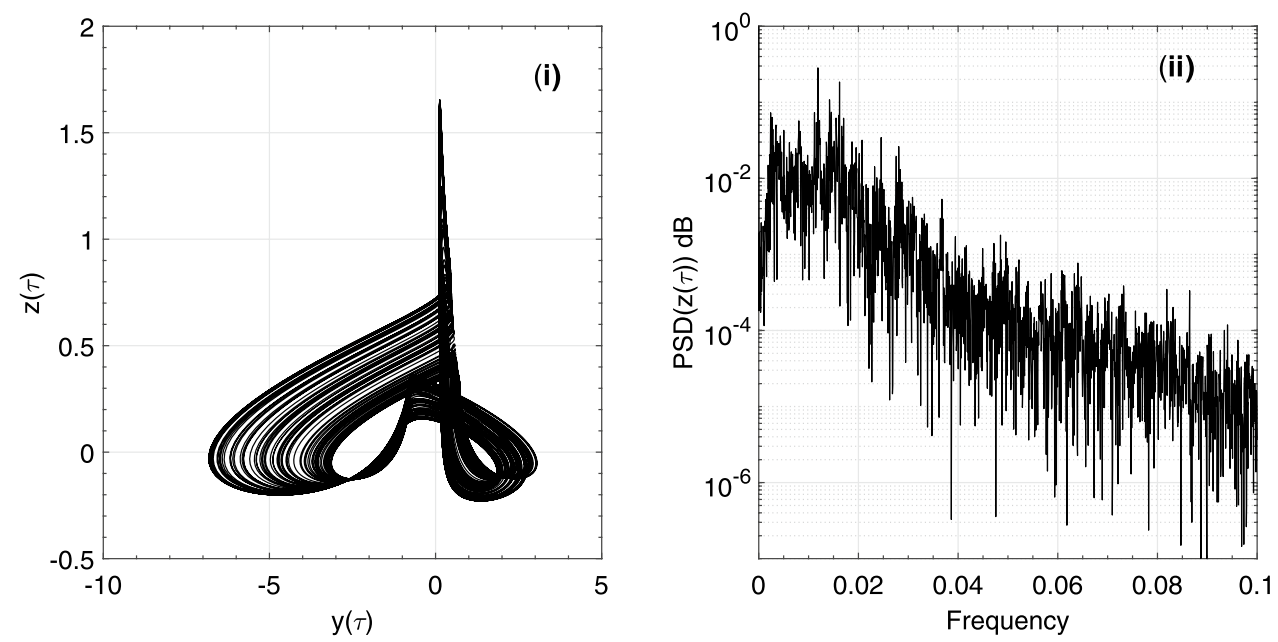

(a)
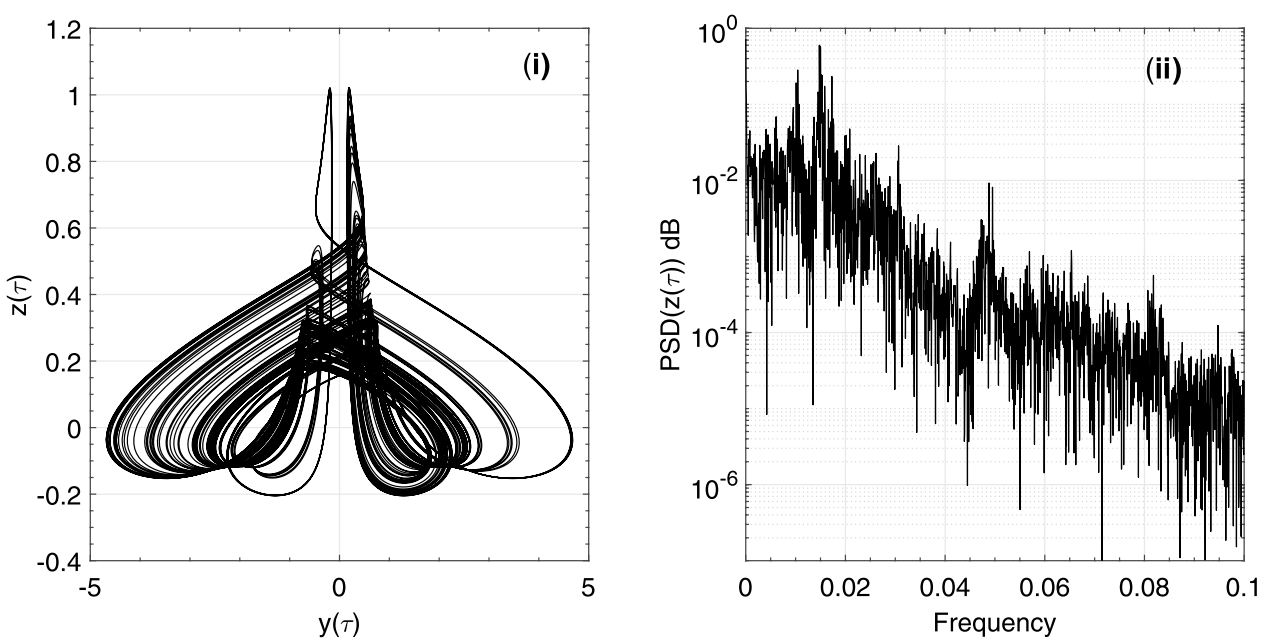

(b)
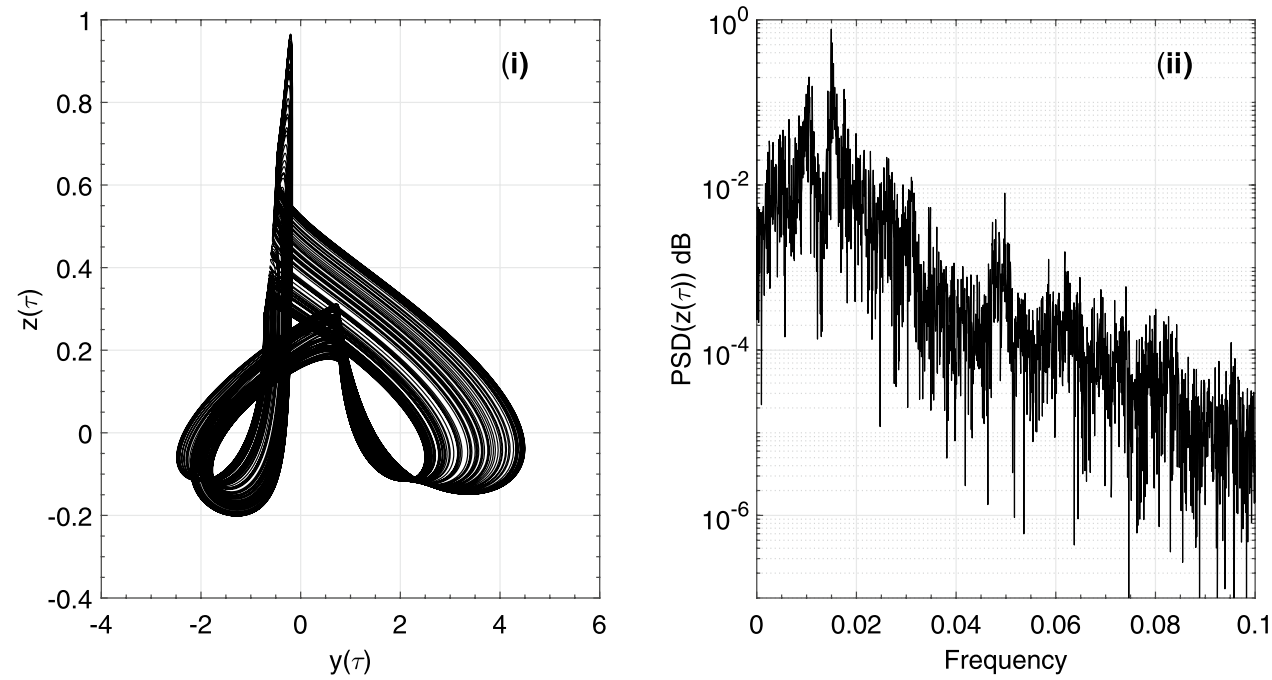

(c) 
confirm these sequences. Finally, interior crisis phenomena sometimes occur in dynamical systems [21, 22, 27], but it is rarely followed by reverse interior crisis. Thus, constitutes a major finding in this recently introduced system by Shahzad et al. [45].

\subsection{Antimonotonicity}

The phenomenon of antimonotonicity was first discovered in nonlinear systems by Dawson et al. [6]. It is described as periodic orbits that can be created and then annihilated via reverse period-doubling bifurcation scenarios as a control parameter is varied. For the system under investigation, the numerically computation of the bifurcation diagrams in Fig. 7 (using $T_{2}$ as a discrete parameter see Fig. 2) illustrates antimonotonicity behavior.
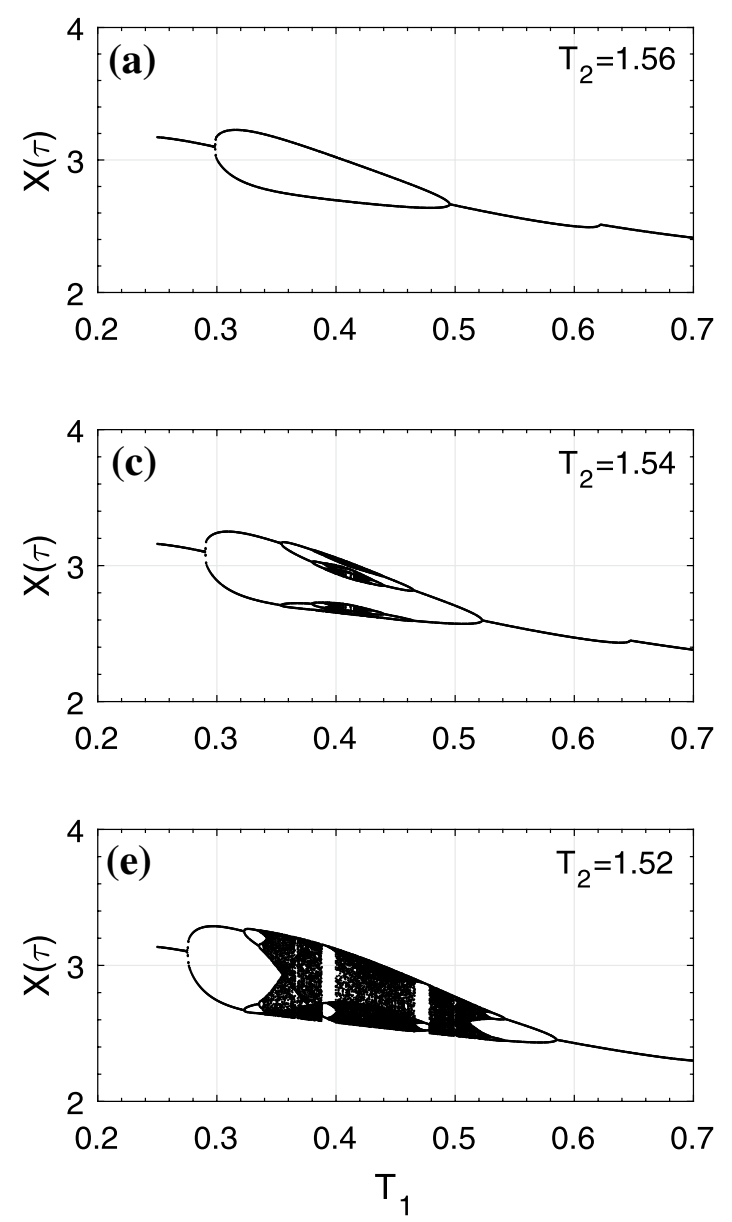

Fig. 7 Bifurcation diagrams illustrating local maxima of the coordinate $x(\tau)$ in terms of control parameter $T_{1}$ showing antimonotonicity phenomenon (bubbles) in system (3): a primary bubble for $T_{2}=1.56$; b period- 4 bubble for $T_{2}=1.55$; c period- 8 bubble
For $T_{2}=1.56$ in Fig. 7 a, a period-2 bubble is observed and the branch develops a stable period- 4 bubble at $T_{2}=1.55$ (see Fig. $7 \mathrm{~b}$ ). As $T_{2}$ is further increased, more bubbles in Fig. 7c-e are created until an infinitely tree (like chaos) finally occurs in Fig. 7f. Accordingly, a first-return map of the local maxima of the coordinate $x(\tau), M_{n+1}[x(\tau)]$ =function $\left(M_{n}[x(\tau)]\right)$ is plotted in Fig. 8a in order to confirm the phenomenon of antimonotonicity in the system described by Eq. (3) and illustrated in Fig. 7.

The map of Fig. 8a is indicative of one-dimensional map with two critical points $P_{1}$ and $P_{2}$ located on each side of the first mediatrix. Therefore, system (3) under investigation according to the results of Dawson exhibits antimonotonicity phenomenon. Accordingly, chaotic hidden bubble attractor are depicted in Fig. $8 \mathrm{~b}-\mathrm{d}$.
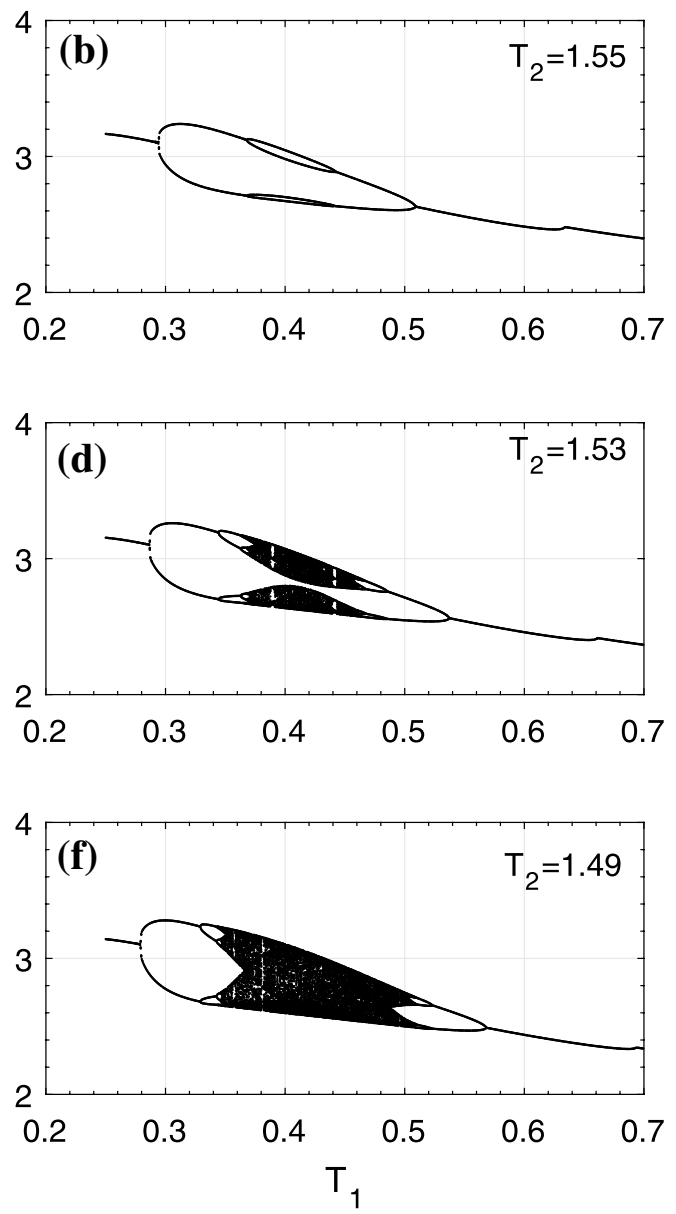

for $T_{2}=1.54 ; \mathbf{d}-\mathbf{f}$ full Feigenbaum remerging tree at $T_{2}=1.53$, $T_{2}=1.52$ and $T_{2}=1.49$, respectively. Parameters are: initial conditions $\left(x_{0} ; y_{0} ; z_{0}\right)=(0.1 ; 0.1 ; 0.1)$ and $T_{1}$ in the range $0.25 \leq T_{1} \leq 0.7$ 
Fig. 8 a First-return map of the local maxima of the coordinate $x(\tau)$ with two critical points $P_{1}$ and $P_{2}$ located on each side of the line of equation firming the phenomenon of antimonotonicity in system (3). b-d 2D phase portraits of chaotic bubbles obtained with the and the parameter $T_{1}=0.42$ $M_{n+1}[x(\tau)]=M_{n}[x(\tau)]$ conparameters of Fig. 7d (bubbles)
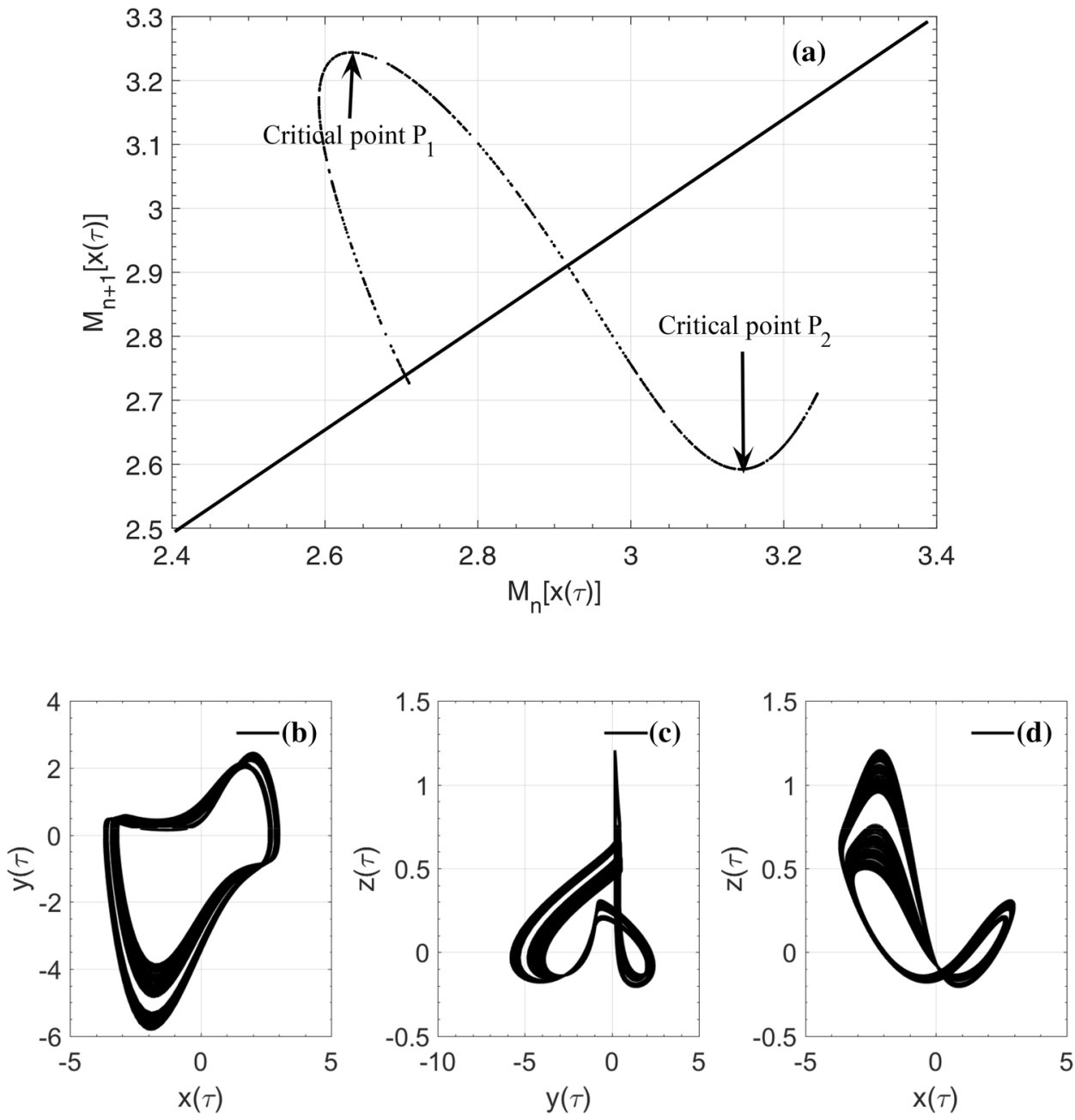

\subsection{Offset boosting and partial amplitude control}

Our goal in this subsection, is to illustrate the flexibility of system (3) to be used as a chaotic encoder circuit using a varying resistor. It could then replace extra expensive amplifier or attenuator in security transmission systems where the chaotic circuit is used as signal source. As in $[32,33]$, we used the re-scaling factor $m \neq 0$ method to control the amplitude of the $x$ and $y$ variables of system (3). To show this, let's insert in system (3) $x \rightarrow x / m, y \rightarrow y / m$, $z \rightarrow z$. The parameter $m$ remains only in the quadratic terms. Then, the amplitude control scheme adjust the size of the attractor only in the $x$ and $y$ axes [according to the third line of Eq. (4)]. The offset boosting control is obtained by replacing $z$ by $(z+k)$ in the second equation of system (4). To this end, parameters $m$ and $k$ are used as control knobs to achieve respectively the offset boot control and partial amplitude goals. The resulting system is as follows.

where $k$ and $m$ are defined above. Figure 9 displays chaotic attractors in (a) $z-x$ plane and (b) in $z-y$ plane.

In Fig. 9, attractors are adjusted in the $z$-axis by means of the control parameter $k$. Interestingly, when increasing $k$ from -0.1 to 2.6 , the location of the attractor in the $z$-axis moves downward from the interval [ -0.11 1.6] (black diagrams) to [-2.8 -1.1] (red diagrams). The average value of the state variables of system (4) versus the control parameter $\mathrm{k}$ is presented in Fig. 10.

Figure 10 shows that the offset controller $k$ will only change the average value of the signal $z$ but will not influence the dynamics of the chaotic system. The phase portraits of system (4) for different values of the control 


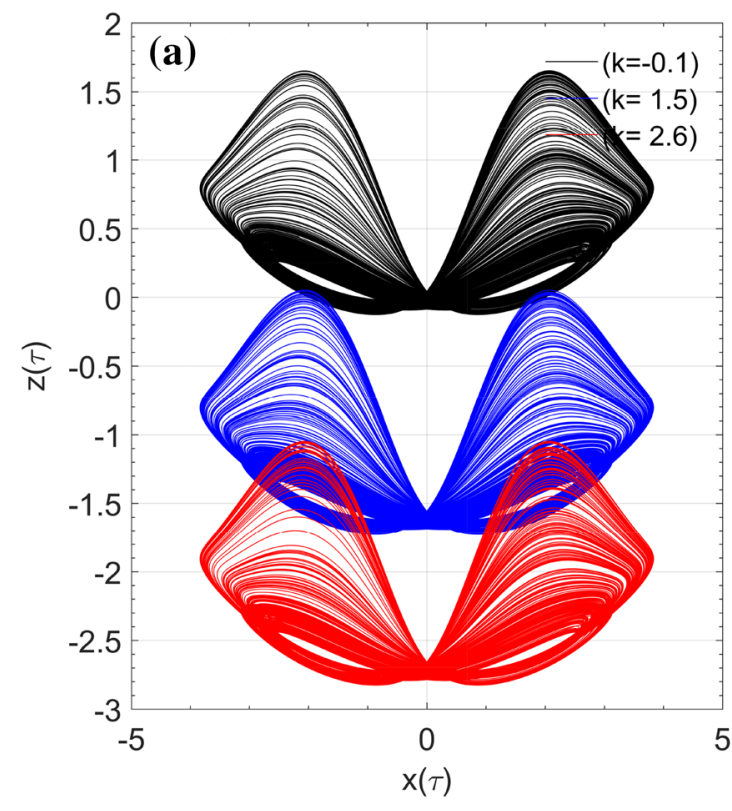

Fig. 9 Chaotic phase portraits of system (4) showing offset boosting dynamics for different values of the control parameter $\mathrm{k}$ : a projection in $z-x$ plane and $\mathbf{b}$ projection in $z-y$ plane. $k=-0.1$ (black); $k=1.5$ (blue) and $k=2.6$ (red). The system parameters are

parameter $m$ are depicted in Fig. 11 confirming the offset boosting in the $x$ and $y$ axes.

In Fig. 11, one can notice that the amplitude of variable $x$ (see Fig. 11a) and $y$ (see Fig. 11b) are stretched or expanded according to the factor $m$. The attractor along the $z$-axis does not change in size because the parameter $m$ in the third equation of system (4) does not affect the variable $z$.

\section{PSIM simulations and experimental results}

Our goal in this section is to verify the offset boosting and the partial amplitude control features by using PSIM and also the bistable chaotic and bubble hidden attractors behaviors using the experimental PCB of the circuit in Fig. 1 b. Therefore, a suitable electronic circuit from mathematical model is designed (4) and illustrated in Fig. 12.

It is easy to see that the resulting circuit is a slightly modification of the circuit in Fig. 1a. The two newly introduced parameters in model (4) are converted as follows: The first parameter $m$ is set using the tuning resistor $R_{m}$ while the second parameter $k$ is set using the new feedback loop containing variable resistor $R_{k}$. Applying

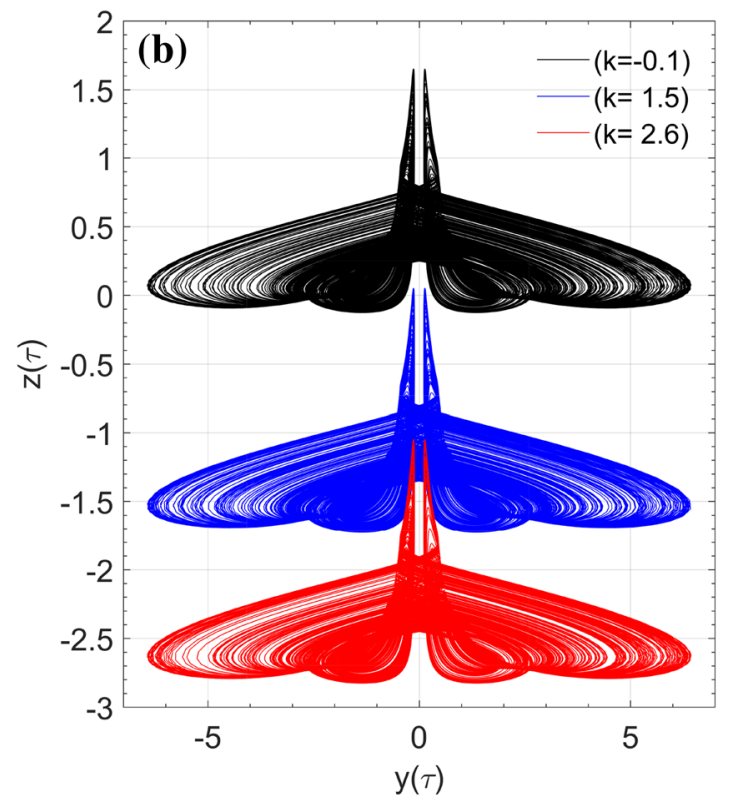

fixed as $m=1, T_{1}=0.41, T_{2}=1.476$ and the initial conditions are selected as $\left(x_{0} ; y_{0} ; z_{0}\right)=(0.1 ; 0.1 ; 0.1)$. (For the interpretation of the references to color in this figure, the reader is referred to the web version of this article)

Kirchhoff's laws to the circuit of Fig. 12, the following set of differential equations (5) are obtained.

$$
\left\{\begin{array}{l}
R C \frac{d V x}{d t}=V y, \\
R C \frac{d V y}{d t}=-V x-10 V y\left(\frac{V z}{1 V}+\frac{R}{R_{k}}\right), \\
R C \frac{d V z}{d t}=\frac{R}{10 R_{m}} \frac{V x V y}{1 V}+\frac{R_{1}}{10 R_{m}} \frac{V x^{2}}{1 V}-\frac{1 V R_{2}}{10 R} .
\end{array}\right.
$$

By setting $R_{k}$ as an open resistor, $R_{m}=R$ and using Eq. (2), the set of three differential equations of Eq. (5) are the same in Eq. (1).

The first confirming result of this section, is the offset boosting of the chaotic attractor in the $V_{z}$ axis shown in Fig. 13a, b.

These figures are obtained by setting the knob resistor $R_{k}$ in the circuit of Fig. 12 as: $R_{k}=10 \mathrm{k} \Omega$, (black curve), $R_{k}=0.66 \mathrm{k} \Omega$ (blue curve) and $R_{k}=0.4 \mathrm{k} \Omega$ (red curve) $\left(R_{k}=R / k\right)$. The reader can verify that in Fig. 13, projections of the resulting attractors in (a) $V z-V x$ plane and in (b) $V z-V y$ plane are similar with numerical results in Fig. 9 of Sect. 3.4.

The second confirming result of this section, is the partial amplitude control feature (see Fig. 13c, d) of the circuit of Fig. 12. The attractor is stretched (or shrunk) along the $V x$ and $V y$ (see Fig. 13c, d) axes by setting the twin knob 

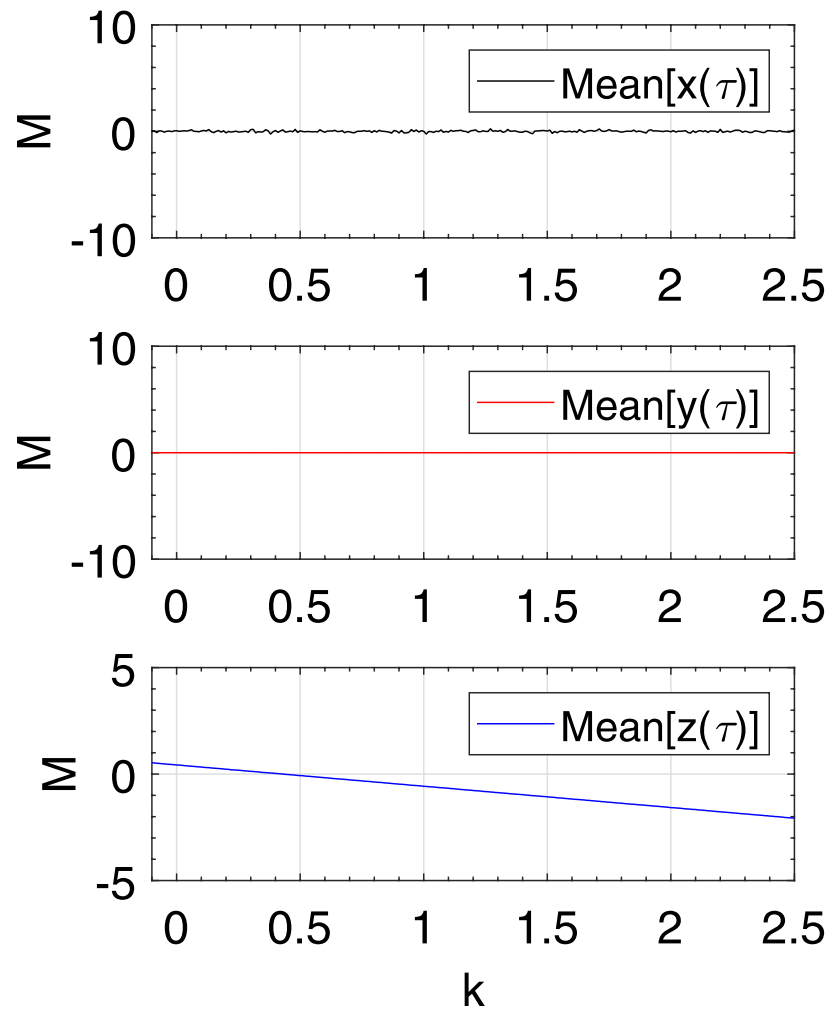

Fig. 10 Mean value of state variables $x(\tau), y(\tau), z(\tau)$ for increasing value of the offset boosting controller, $k \in[-0.1,2.5]$ displaying the offset boosting dynamics in system (4). The initial conditions setting are $\left(x_{0} ; y_{0} ; z_{0}\right)=(0.1 ; 0.1 ; 0.1)$. (For the interpretation of the references to color in this figure, the reader is referred to the web version of this article)

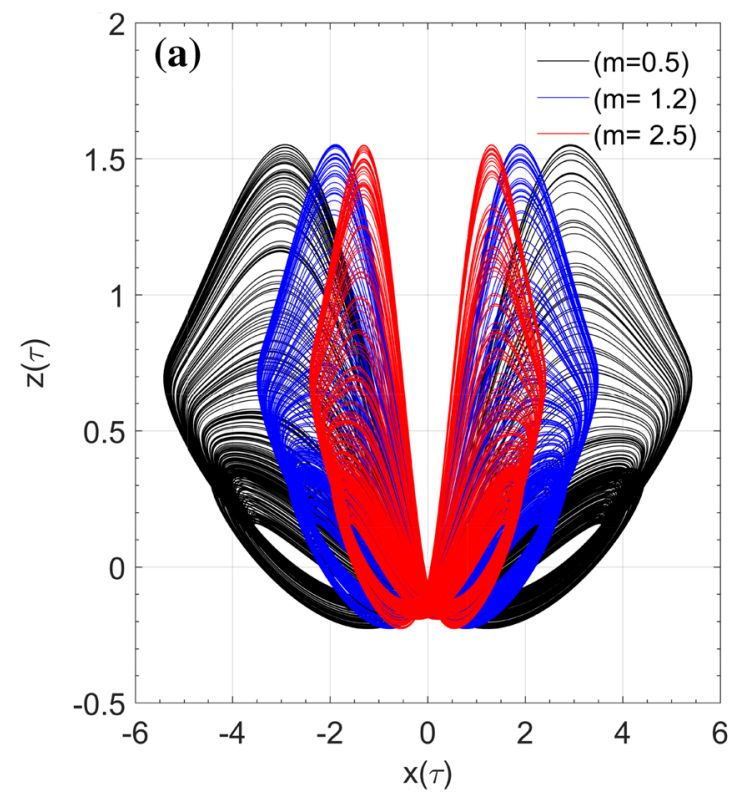

Fig. 11 Amplitude control of chaotic attractors of system (4) for different values of the parameters $m$ : a projection in $z-x$ plane and $\mathbf{b}$ projection in $z-y$ plane. $m=0.5$ (black); $m=1.2$ (blue) and $m=2.5$ (red). The rest of the parameters are fixed resistors $R_{m^{\prime}}\left(R_{m}=R / m\right)$ of circuit in Fig. 12 to $R_{m}=200 \mathrm{k} \Omega$ (black curve), $R_{m}=12 \mathrm{k} \Omega$ (blue curve) and $R_{m}=50 \mathrm{k} \Omega$ (red curve):: (c) Projection in $V z-V x$ plane and (d) projection in $V z-V y$ plane: The resistor $R_{k}=\infty$ (open loop). There are good agreements with numerical results in Fig. 11 of the numerical Sect. 3.4.

The experimental results captured on the PCB, display in Figs. 14 and 15 confirmed the chaotic bistable and chaotic bubble hidden attractors in the circuit respectively.

The DC voltage is tuned to $1.59 \mathrm{~V}$, the first resistor $R_{2}$ to $10 \mathrm{k} \Omega$ and the second resistor $R_{1}$ is slowly adjusted from 2 to $6 \mathrm{k} \Omega$. The Fig. $14 \mathrm{a}-\mathrm{c}$ show $2 \mathrm{D}$ phase portraits of chaotic bistability behavior of the circuit. The first line of figures resembles the black attractors in Fig. 4 while the second line of figures resembles the red attractors in Fig. 4.

The experimental results of the chaotic bubbles hidden attractors are display in Fig. 15.

While slightly adjusting resistor $R_{2}$ around $15 \mathrm{k} \Omega$, two chaotic bubbles appear on the $2 \mathrm{D}$ phase portraits as illustrated in Fig. 15a-c confirming theoretical results in Fig. 8.

It can be notice that the measured values of the resistor $R_{1}$ and $R_{2}$ are slightly different from those that are in the theoretical analysis. This is always due to the assumption made to different electronic components in the theoretical section (ideal operational amplifier, zero tolerance on the resistor value, non-ideal capacitor).

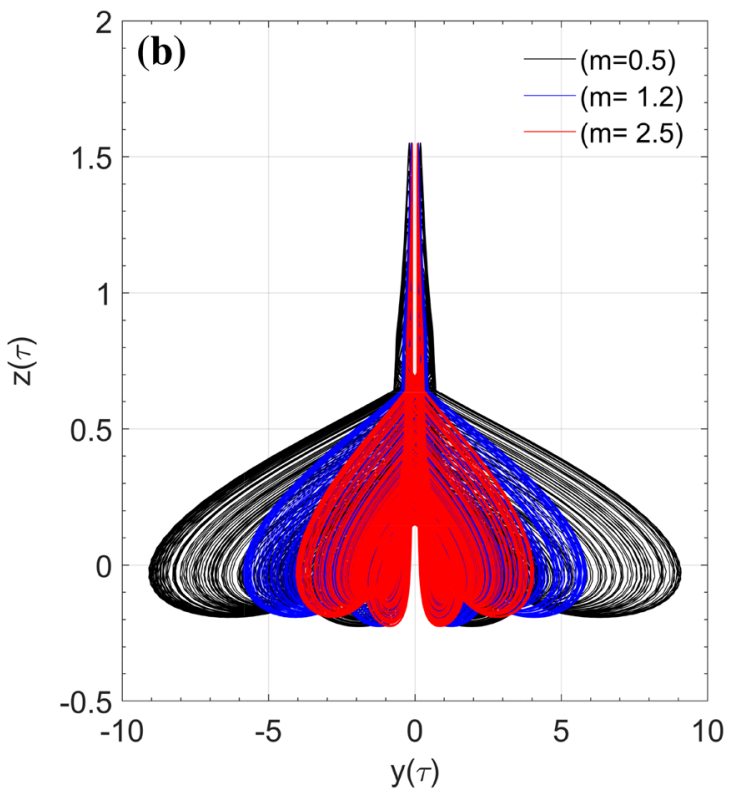

as: $T_{1}=0.41, T_{2}=1.476, k=0$ and the initial conditions are $\left(x_{0} ; y_{0} ; z_{0}\right)=(0.1 ; 0.1 ; 0.1)$. (For the interpretation of the references to color in this figure, the reader is referred to the web version of this article) 


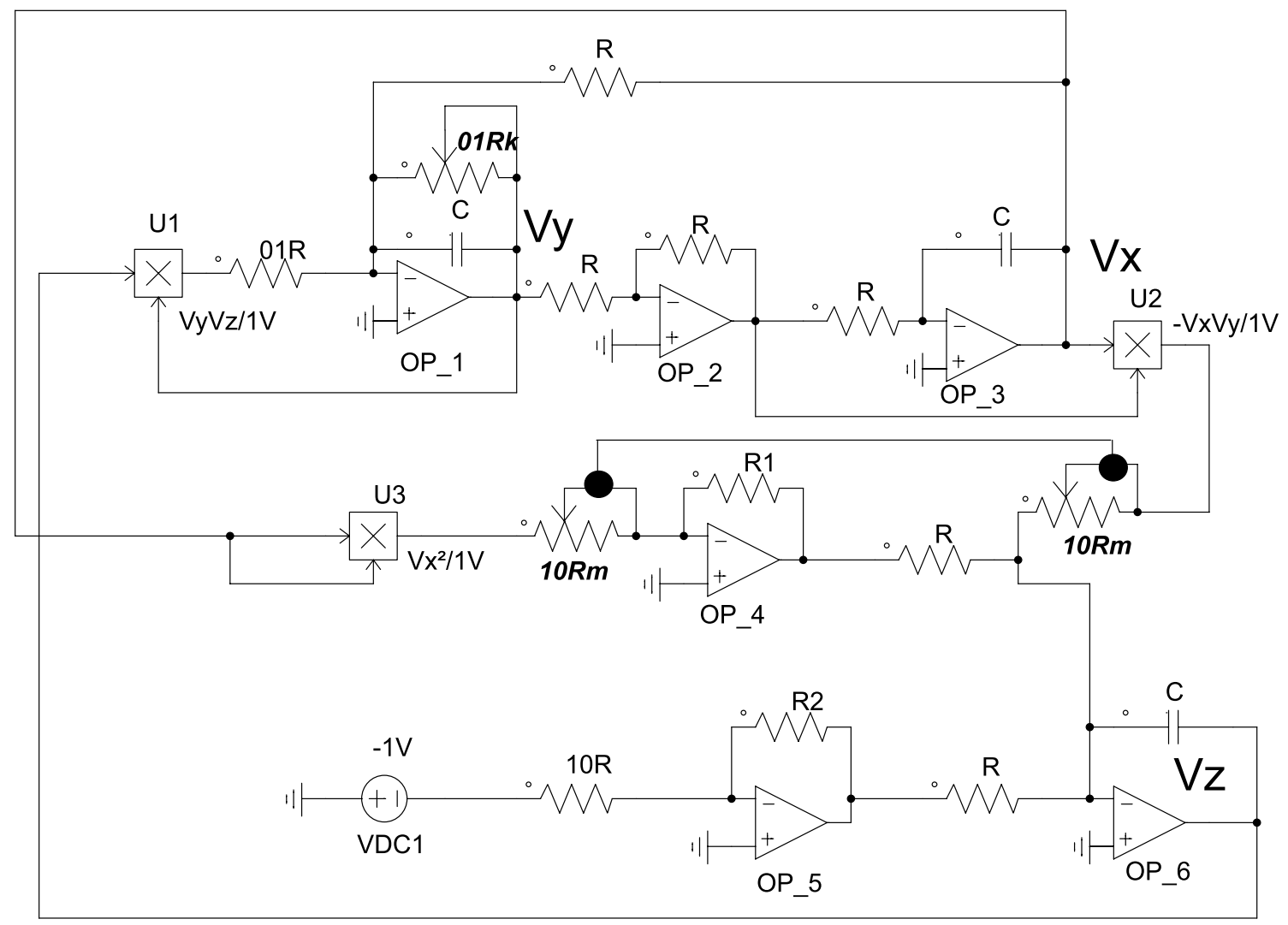

Fig. 12 Electronic circuit implementation of the model (4) obtained by adding variables resistors $R_{k}$ and $R_{m}$ to the circuit in Fig. (1). $R_{k}$ and $R_{m}$ variable resistors. $R_{1}=4.1 \mathrm{k} \Omega$ and $R_{2}=14.76 \mathrm{k} \Omega$. The dark dots stand for simultaneous adjustment of resistor $10 R_{m}$

\section{Conclusion}

In this paper, an electronic circuit modelling the dynamics of a chaotic system without equilibrium point recently introduced by Shahzad et al. [45] was investigated using conventional nonlinear dynamic methods and algorithms. This study shows that the circuit displays new dynamical behaviors, including antimonotonicity, bistable chaotic hidden attractors, chaotic hidden bubbles attractors, interiors crisis (reverse and forward) and offset boosting and partial amplitude control. The bistable and bubble chaotic hidden attractors were illustrated experimentally using the electronic circuit realized while the offset boosting and partial amplitude control were verified using PSIM simulations. An electronic circuit describing the chaotic system without equilibrium point is realized. The observed dynamical behaviors are consistent with those revealed numerically. We are convinced that the results obtained in this paper complete the ones in [45], and enrich the literature with the interesting behaviors of the NE8 no equilibrium system. The chaos-based firefly algorithms $[8,52]$, will be use in future works to outline promising avenues in the system rate equations of the circuit under investigation. 


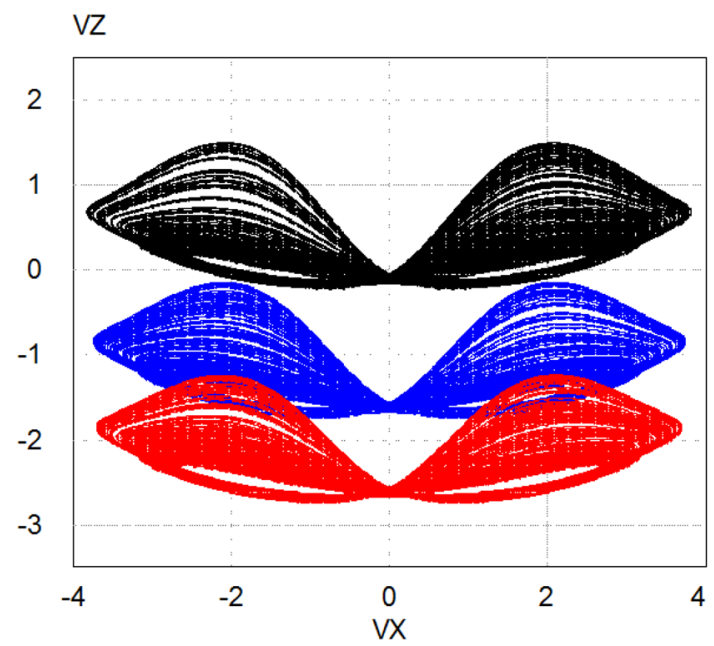

(a)

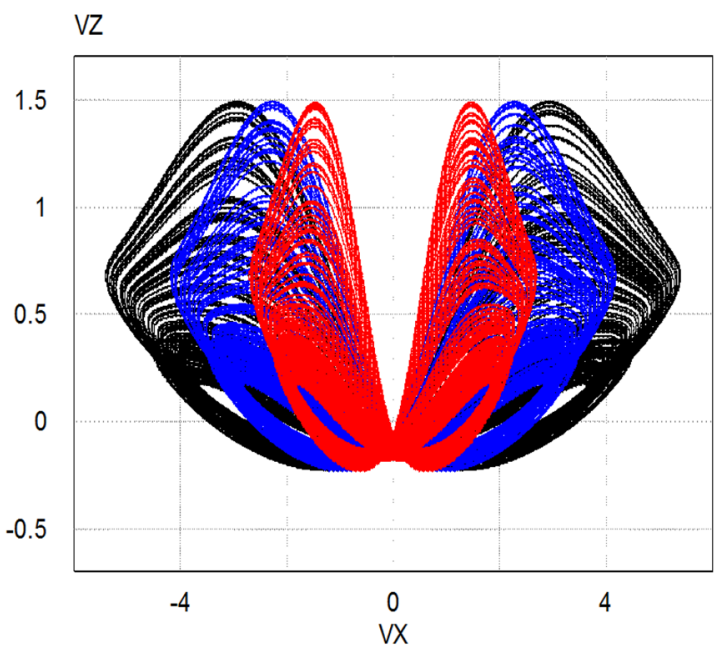

(c)

Fig. 13 Chaotic phase portraits of the circuit of Fig. 12 showing: (first line of figures) offset boosting dynamics for different values of the variable resistor $R_{k}$. $\left(R_{k}=R / k\right)$ : a projection in $V z-V x$ plane and $\mathbf{b}$ projection in $V z-V y$ plane: $R_{k}=10 \mathrm{k} \Omega$ (black); $R_{k}=0.66 \mathrm{k} \Omega$ (blue) and $R_{k}=0.4 \mathrm{k} \Omega$ (red). The circuit resistor $R_{m}$ is set as $R_{m}=100 \mathrm{k} \Omega$. (second line of figures) Amplitude control dynamics for different values of the variable resistor $R_{m}$. $\left(R_{m}=R / m\right)$ : c

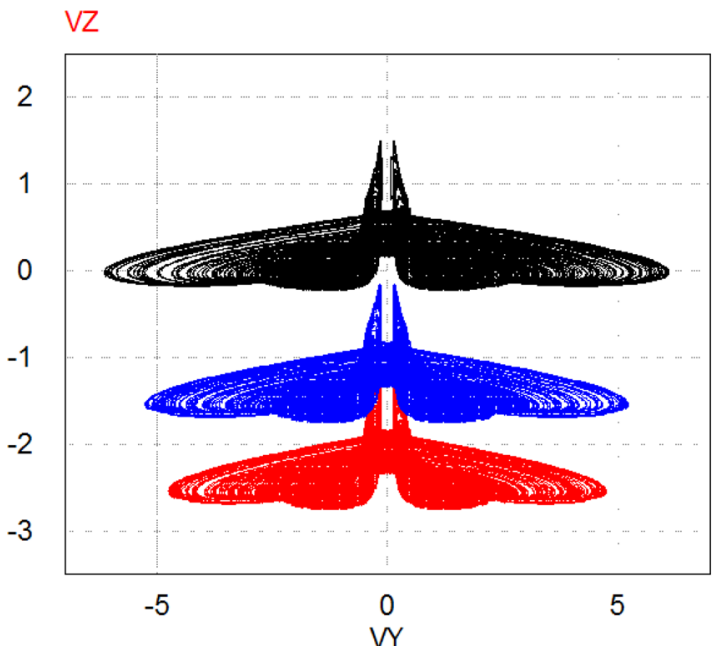

(b)

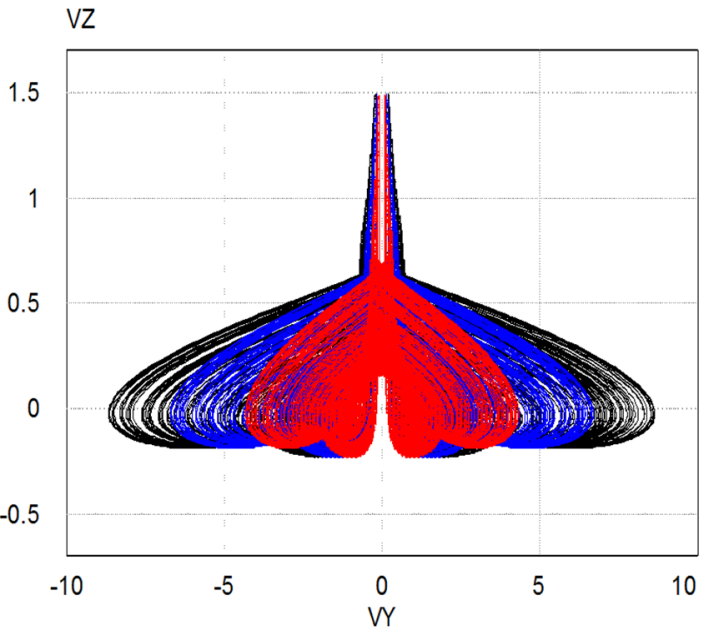

(d)

projection in $V z-V x$ plane and $\mathbf{d}$ projection in $V z-V y$ plane: $R_{m}=200 \mathrm{k} \Omega$ (black); $R_{m}=12 \mathrm{k} \Omega$ (blue) and $R_{m}=50 \mathrm{k} \Omega$ (red). The circuit resistor $R_{k}$ is $R_{k}=\infty$ (open loop). Initial conditions $\left(V x_{0} ; V y_{0} ; V z_{0}\right)=(0.001 \mathrm{~V} ; 3.9 \mathrm{~V} ; 0.1 \mathrm{~V})$. (For the interpretation of the references to color in this figure, the reader is referred to the web version of this article) 

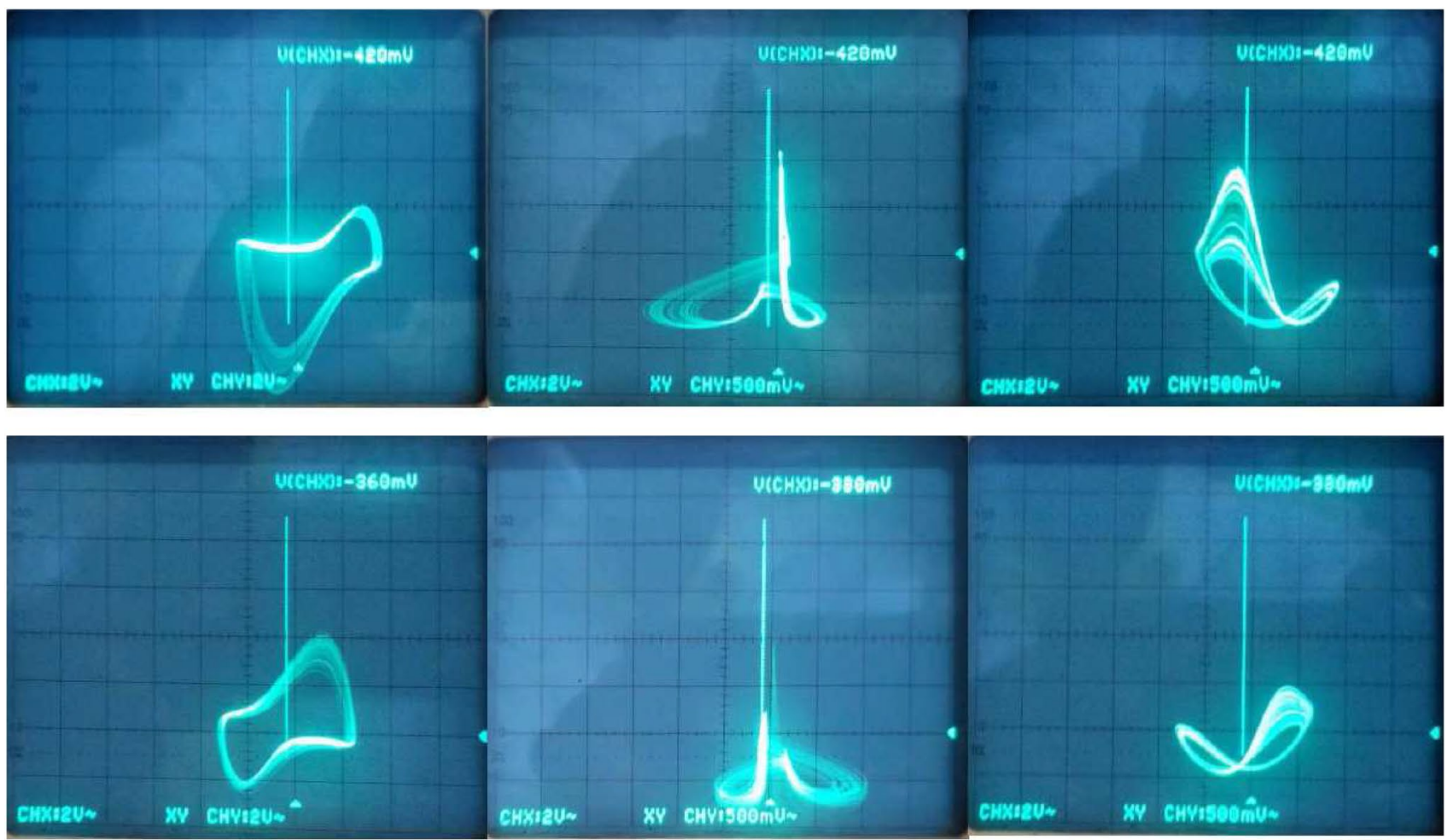

(a)

(b)

(c)

Fig. 14 Chaotic phase portraits captured on PCB confirming the bistability(bistable chaotic hidden attractors) behavior in the circuit of Fig. $1 \mathrm{~b}$ under investigation in the planes $\mathbf{a} V x-V y, \mathbf{b}$
$V y-V z, c V x-V z$. This figure reproduces Fig. 4. Resistors value are $R_{2}=14.76 \mathrm{k} \Omega$ and $R_{1}=0.395 \mathrm{k} \Omega$

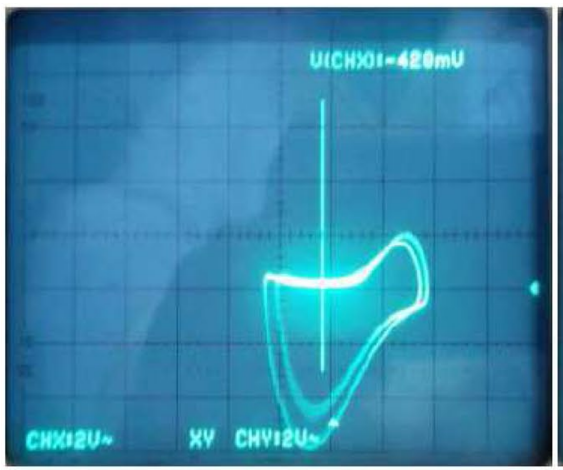

(a)

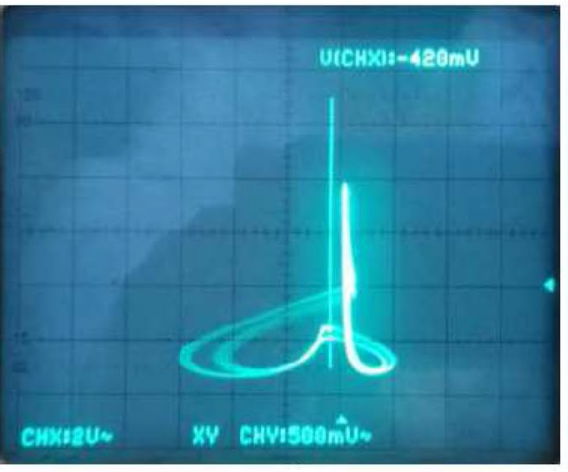

(b)

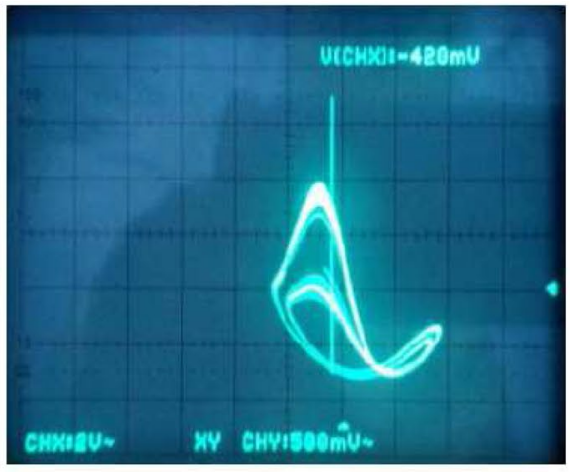

(c)
Fig. 15 chaotic phase portraits captured on PCB confirming the phenomenon of antimonotonicity (chaotic bubble hidden attractors) in the circuit (in Fig. 1b) in the planes a $V x-V y, \mathbf{b} V y-V z, \mathbf{c}$

Acknowledgements The authors would like to thank Dr. Sifeu Takougang Kingni (University of Maroua, Cameroon), for carefully reading the manuscript and Prof. Jacques Kengne (IUT-FV of University of Dschang, Cameroon) for many helpful pieces of advice during the
$V x-V z$. These figures reproduce Fig. $8 \mathrm{~b}-\mathrm{d}$. Resistors value are: $R_{2}=15.35 \mathrm{k} \Omega$ and $R_{1}=4.2 \mathrm{k} \Omega$

investigation of this work. The authors thank the anonymous reviewers for their many insightful comments and suggestions who help to improve the quality of this paper. 


\section{Compliance with ethical standards}

Conflict of interest On behalf of all the authors, the corresponding author declares that there is no conflict of interest in this research article.

\section{References}

1. Andrievsky B, Kuznetsov N, Leonov G, Pogromsky A (2013) Hidden oscillations in aircraft flight control system with input saturation. IFAC Proc Vol 46(12):75-79

2. Argyris JH, Faust G, Haase M, Friedrich R (2015) An exploration of dynamical systems and chaos: completely revised and enlarged, 2nd edn. Springer, New York

3. Bao G, Zeng Z (2013) Multistability of periodic delayed recurrent neural network with memristors. Neural Comput Appl 23(7):1963-1967

4. Blazejczyk-Okolewska B, Kapitaniak T (1996) Dynamics of impact oscillator with dry friction. Chaos Solitons Fractals 7(9):1455-1459

5. Boriga R, Dafscaflescu AC, Priescu I (2014) A new hyperchaotic map and its application in an image encryption scheme. Signal Process Image Commun 29(8):887-901

6. Dawson S, Grebogi C, Yorke J, Kan I, Koçak H (1992) Antimonotonicity: inevitable reversals of period-doubling cascades. Phys Lett A 162(3):249-254

7. Filali RL, Benrejeb M, Borne P (2014) On observer-based secure communication design using discrete-time hyperchaotic systems. Commun Nonlinear Sci Numer Simul 19(5):1424-1432

8. Fister IJ, Perc M, Kamal SM, Fister I (2015) A review of chaos-based firefly algorithms: perspectives and research challenges. Appl Math Comput 252:155-165. https://doi. org/10.1016/j.amc.2014.12.006

9. Ginoux JM, Ruskeepaa H, Perc M, Naeck R, Costanzo VD, Bouchouicha M, Fnaiech F, Sayadi M, Hamdi T (2018) Is type 1 diabetes a chaotic phenomenon? Chaos Solitons Fractals 111:198-205. https://doi.org/10.1016/j.chaos.2018.03.033

10. Grebogi C, Ott E, Yorke J (1982) Chaotic attractors in crisis. Phys Rev Lett 48(22):1507-1510

11. Grebogi C, Ott E, Yorke J (1987) Basin boundary metamorphoses: changes in accessible boundary orbits. Nucl Phys B Proc Suppl 2:281-300. https://doi.org/10.1016/09205632(87)90024-7

12. Hao Z, Cao Q (2014) A novel dynamical model for gvt nonlinear supporting system with stable-quasi-zero-stiffness. J Theor Appl Mech 52(1):199-213

13. Hasler M, Maistrenko Y (1997) An introduction to the synchronization of chaotic systems: coupled skew tent maps. IEEE Trans Circuits Syst I Fundam Theory Appl 44(10):856-866

14. Healey J, Broomhead D, Cliffe K, Jones R, Mullin T (1991) The origins of chaos in a modified van der pol oscillator. Physica $D$ 48(2):322-339

15. Hu X, Liu C, Liu L, Ni J, Li S (2016) Multi-scroll hidden attractors in improved sprott a system. Nonlinear Dyn 86(3):1725-1734

16. Jafari S, Sprott J, Golpayegani S (2013) Elementary quadratic chaotic flows with no equilibria. Phys Lett A 377(9):699-702

17. Jafari S, Pham VT, Kapitaniak T (2016) Multiscroll chaotic sea obtained from a simple 3D system without equilibrium. Int J Bifurc Chaos 26(02):1650031-1650038

18. Kaddoum $G$ (2016) Wireless chaos-based communication systems: a comprehensive survey. IEEE Access 4:2621-2648. https ://doi.org/10.1109/ACCESS.2016.2572730
19. Kahn J, Kapitaniak B, Monod H (1985) Comparison of two modalities when exerting isometric contractions. Eur J Appl Physiol 54(3):331-335

20. Kamdoum Tamba V, Fotsin H, Kengne J, Megam Ngouonkadi $E$, Talla P (2017) Emergence of complex dynamical behaviors in improved colpitts oscillators: antimonotonicity, coexisting attractors, and metastable chaos. Int J Dyn Control 5(3):395-406

21. Kengne J, Chedjou J, Kom M, Kyamakya K, Tamba VK (2014) Regular oscillations, chaos, and multistability in a system of two coupled van der pol oscillators: numerical and experimental studies. Nonlinear Dyn 76(2):1119-1132

22. Kengne J, Njitacke Z, Nguomkam Negou A, Fouodji Tsostop M, Fotsin $\mathrm{H}$ (2016) Coexistence of multiple attractors and crisis route to chaos in a novel chaotic jerk circuit. Int J Bifurc Chaos 26(5):1650081-16500100

23. Khajanchi S, Perc M, Ghosh D (2018) The influence of time delay in a chaotic cancer model. Chaos Interdiscip J Nonlinear Sci 28(10):103101

24. Khan A, Budhraja M, Ibraheem A (2018) Multi-switching dual compound synchronization of chaotic systems. Chin J Phys 56(1):171-179

25. Kingni S, Jafari S, Simo H, Woafo P (2014) Three-dimensional chaotic autonomous system with only one stable equilibrium: analysis, circuit design, parameter estimation, control, synchronization and its fractional-order form. Eur Phys J Plus 129(5):76-82

26. Koyuncu I, Turan Özcerit A (2016) The design and realization of a new high speed FPGA-based chaotic true random number generator. Comput Electr Eng 58:203-214. https://doi.org/10.1016/j. compeleceng.2016.07.005

27. Kuznetsov A, Kuznetsov S, Mosekilde E, Stankevich N (2015) Coexisting hidden attractors in a radio-physical oscillator system. J Phys A Math Theor 48(12):125101-1251013

28. Kuznetsov N, Leonov G, Vagaitsev V (2010) Analytical-numerical method for attractor localization of generalized Chua's system. IFAC Proc 43(11):29-33

29. Leonov G, Kuznetsov N (2013) Hidden attractors in dynamical systems. from hidden oscillations in hilbert-kolmogorov, aizerman, and kalman problems to hidden chaotic attractor in chua circuits. Int J Bifurc Chaos 23(01):1330002-1330012

30. Leonov G, Kuznetsov N, Vagaitsev V (2012) Hidden attractor in smooth chua systems. Physica D 241(18):1482-1486

31. Leonov G, Kuznetsov N, Mokaev T (2015) Homoclinic orbits, and self-excited and hidden attractors in a lorenz-like system describing convective fluid motion. Eur Phys J Spec Top 224(8):1421-1458

32. Li C, Sprott J (2014) Chaotic flows with a single nonquadratic term. Phys Lett A 378(3):178-183

33. Li C, Sprott J (2016) Variable-boostable chaotic flows. Opt Int J Light Electron Opt 127(22):10389-10398

34. Li XZ, Chan SC (2013) Heterodyne random bit generation using an optically injected semiconductor laser in chaos. IEEE J Quantum Electron 49(10):829-838

35. Molaie M, Jafari S, Sprott J, Golpayegani S (2013) Simple chaotic flows with one stable equilibrium. Int J Bifurc Chaos 23(11):1350188-13501

36. Perc $M(2005)$ Visualizing the attraction of strange attractors. Eur J Phys 26(4):579-587

37. Pham VT, Jafari S, Volos C, Wang X, Golpayegani S (2014a) Is that really hidden? The presence of complex fixedpoints in chaotic flows with no equilibria. Int J Bifurc Chaos 24(11):1450146-145022

38. Pham VT, Volos C, Jafari S, Wei Z, Wang X (2014b) Constructing a novel no-equilibrium chaotic system. Int J Bifurc Chaos 24(05):1450073-1450081

39. Pham VT, Volos CK, Vaidyanathan S (2015) Multi-scroll chaotic oscillator based on a first-order delay differential equation. 
In: Azar AT, Vaidyanathan S (eds) Chaos modeling and control systems design, Studies in computational intelligence, vol 581. Springer, Cham

40. Pisarchik A, Feudel U (2014) Control of multistability. Phys Rep 540(4):167-218

41. Posch H, Hoover W, Vesely F (1986) Canonical dynamics of the nosé oscillator: stability, order, and chaos. Phys Rev A 33(6):4253-4266

42. Pribylova L (2009) Bifurcation routes to chaos in an extended Van der Pol's equation applied to economic models. Electron J Differ Equ 2009(52):1-21

43. Ren HP, Bai C, Liu J, Baptista M, Grebogi C (2016) Experimental validation of wireless communication with chaos. Chaos Interdiscip J Nonlinear Sci 26(8):083117-083126

44. Shafer D (1995) Nonlinear dynamics and chaos: with applications to physics, biology, chemistry, and engineering (steven $\mathrm{h}$. strogatz). SIAM Rev 37(2):280-281

45. Shahzad M, Pham VT, Ahmad M, Jafari S, Hadaeghi F (2015) Synchronization and circuit design of a chaotic system with coexisting hidden attractors. Eur Phys J Spec Top 224(8):1637-1652

46. Silchenko A, KapitaniakT, Anishchenko V (1999) Noise-enhanced phase locking in a stochastic bistable system driven by a chaotic signal. Phys Rev E 59(2):1593-15100

47. Sprott J, Hoover W, Hoover C (2014) Heat conduction, and the lack thereof, in time-reversible dynamical systems: generalized nosé-hoover oscillators with a temperature gradient. Phys Rev E 89(4):042914-042929

48. Swathy PS, Thamilmaran K (2014) Hyperchaos in SC-CNN based modified canonical Chua's circuit. Nonlinear Dyn 78(4):2639-2650

49. Tahir F, Jafari S, Pham VT, Volos C, Wang X (2015) A novel noequilibrium chaotic system with multiwing butterfly attractors. Int J Bifurc Chaos 25(04):1550056

50. Tlelo-Cuautle E, De La Fraga L, Pham VT, Volos C, Jafari S, Quintas-Valles AdJ (2017) Dynamics, FPGA realization and application of a chaotic system with an infinite number of equilibrium points. Nonlinear Dyn 89(2):1129-1139

51. Vaidyanathan S, Volos C, Pham VT, Madhavan K, Idowu B (2014) Adaptive backstepping control, synchronization and circuit simulation of a 3-D novel jerk chaotic system with two hyperbolic sinusoidal nonlinearities. Arch Control Sci 24(3):375-403

52. Wang H, Wang W, Zhou X, Sun H, Zhao J, Yu X, Cui Z (2017a) Firefly algorithm with neighborhood attraction. Inf Sci 382-383:374-387

53. Wang Z, Akgul A, Pham VT, Jafari S (2017b) Chaos-based application of a novel no-equilibrium chaotic system with coexisting attractors. Nonlinear Dyn 89(3):1877-1887

54. Wei Z, Bin Z, Yang J, Perc M, Slavinec M (2019) Bifurcation analysis of two disc dynamos with viscous friction and multiple time delays. Appl Math Comput 347:265-281. https://doi. org/10.1016/j.amc.2018.10.090

55. Wolf A, Swift J, Swinney H, Vastano J (1985) Determining lyapunov exponents from a time series. Physica D 16(3):285-317

56. Zeng Z, Zheng W (2012) Multistability of neural networks with time-varying delays and concave-convex characteristics. IEEE Trans Neural Netw Learn Syst 23(2):293-305

Publisher's Note Springer Nature remains neutral with regard to jurisdictional claims in published maps and institutional affiliations. 